\title{
Comments on: Stability in linear optimization and related topics. A personal tour
}

\author{
Klatte, Diethard
}

\begin{abstract}
This is a well-written compendium of both classical and recent results on qualitative and quantitative stability of linear optimization problems, where the case of infinitely many constraints is intrinsically included. The author presents basic theoretical approaches and outcomes of this area in a concise, competent and informative manner. Since this survey is almost complete, we cannot add much in our note. So, we will restrict ourselves to a discussion of two special subjects which are related to our own research, complementing thereby single aspects of the author's presentation of Lipschitz stability analysis.
\end{abstract}

DOI: https://doi.org/10.1007/s11750-011-0217-5

Other titles: Discussion: "Stability in linear optimization and related topics. A personal tour"

Posted at the Zurich Open Repository and Archive, University of Zurich

ZORA URL: https://doi.org/10.5167/uzh-67234

Journal Article

Originally published at:

Klatte, Diethard (2012). Comments on: Stability in linear optimization and related topics. A personal tour. Top. An Official Journal of the Spanish Society of Statistics and Operations Research, 20(2):250254.

DOI: https://doi.org/10.1007/s11750-011-0217-5 


\title{
Discussion: "Stability in linear optimization and related topics. A personal tour"
}

\author{
Diethard Klatte
}




\title{
Discussion: "Stability in Linear Optimization and Related Topics. A Personal Tour" by Marco A. López
}

\author{
Diethard Klatte ${ }^{1}$ \\ 18 April 2011
}

This is a well-written compendium of both classical and recent results on qualitative and quantitative stability of linear optimization problems, where the case of infinitely many constraints is intrinsically included. The author presents basic theoretical approaches and outcomes of this area in a concise, competent and informative manner. Since this survey is almost complete, we cannot add much in our note. So, we will restrict ourselves to a discussion of two special subjects which are related to our own research, complementing thereby single aspects of the author's presentation of Lipschitz stability analysis.

\section{Sharp Lipschitz constants for finite linear systems}

The author is particularly interested in concrete and computable formulae for local and global error bounds in the sense of his definitions (1.2), (1.3) and (1.4). For systems of finitely many linear equations and inequalities, there is a related classical subject: the study of (sharp) Lipschitz or Hoffman constants. However, under right-hand side perturbations of finite linear systems, Lipschitz continuity of the solution set map is uniform. So one is also interested in uniform constants.

To give some details, let $A$ and $C$ be real matrices of dimensions $m \times n$ and $k \times n$, respectively, define a multifunction by

$$
\mathcal{F}(b, d):=\left\{x \in \mathbb{R}^{n} \mid A x \leq b, C x=d\right\}, \quad(b, d) \in \mathbb{R}^{m} \times \mathbb{R}^{k},
$$

and denote by $\operatorname{dom} \mathcal{F}$ its effective domain. For $X, Y \subset \mathbb{R}^{n}$ and a given norm $\|\cdot\|_{\beta}$, let $e_{\beta}(X, Y)=\sup _{x \in X} \inf _{y \in Y}\|y-x\|_{\beta}$ be the HausdorffPompeiu excess of $X$ over $Y$, with $e_{\beta}(\emptyset, Y):=0$. By Hoffman's (1952) fundamental theorem, there exists a constant $\varrho_{\alpha \beta}=\varrho_{\alpha \beta}(A, C)>0$, called Lipschitz constant for $\mathcal{F}$, such that for all $(b, d),\left(b^{\prime}, d^{\prime}\right) \in \operatorname{dom} \mathcal{F}$,

$$
e_{\beta}\left(\mathcal{F}\left(b^{\prime}, d^{\prime}\right), \mathcal{F}(b, d)\right) \leq \varrho_{\alpha \beta}\left\|\left(\begin{array}{c}
b-b^{\prime} \\
d-d^{\prime}
\end{array}\right)\right\|_{\alpha}
$$

\footnotetext{
${ }^{1}$ Address: IBW, Universität Zürich, Moussonstrasse 15, CH-8044 Zürich, Switzerland. E-Mail: diethard.klatte@business.uzh.ch
} 
where $\|\cdot\|_{\alpha}$ and $\|\cdot\|_{\beta}$ are any two norms in $\mathbb{R}^{m+k}$ and $\mathbb{R}^{n}$, respectively. This means that the multifunction $\mathcal{F}$ is Lipschitz on $\operatorname{dom} \mathcal{F}$ with respect to the related Hausdorff metric $h_{\beta}(X, Y)=\max \left\{e_{\beta}(X, Y), e_{\beta}(Y, X)\right\}$ for convex polyhedral sets $X, Y$. A constant $\varrho_{\alpha \beta}$ for $\mathcal{F}$ is called sharp if there exist $(b, d),\left(b^{\prime}, d^{\prime}\right) \in \operatorname{dom} \mathcal{F}$ such that $h_{\beta}\left(\mathcal{F}\left(b^{\prime}, d^{\prime}\right), \mathcal{F}(b, d)\right) \geq$ $\varrho_{\alpha \beta}\left\|\left(\begin{array}{c}b-b^{\prime} \\ d-d^{\prime}\end{array}\right)\right\|_{\alpha}>0$.

Obviously, any Lipschitz constant $\varrho_{\alpha \beta}$ also satisfies the original version of Hoffman's Lemma (1952), namely, for $(b, d) \in \operatorname{dom} \mathcal{F}$,

$$
\inf _{y \in \mathcal{F}(b, d)}\|y-x\|_{\beta} \leq \varrho_{\alpha \beta}\left\|\begin{array}{l}
(A x-b)_{+} \\
(C x-d)
\end{array}\right\|_{\alpha} \quad \forall x \in \mathbb{R}^{n},
$$

so $\varrho_{\alpha \beta}$ is often called a Hoffman constant for $\mathcal{F}$. Note that (3) implies (2), provided that $\|\cdot\|_{\alpha}$ is a monotonic norm (i.e., $\|z\|_{\alpha}=\||z|\|_{\alpha}$ ), cf. Mangasarian and Shiau (1986). For other types of monotonicity for $\|\cdot\|_{\alpha}$ in relating (3) and (2), see Zalinescu (2003).

If (2) (or (3)) holds for fixed $(b, d) \in \operatorname{dom} \mathcal{F}$ with a constant $\varrho_{\alpha \beta}$ depending on $(A, C, b, d)$, one speaks of a Lipschitz (Hoffman) constant for $\mathcal{F}$ at $(b, d)$. This notion corresponds to the author's definition (1.4). Inspired by the big progress of studying quantitative stability for nonlinear systems in the 1990ies, several authors applied the related ideas to linear systems, in particular, for deriving (sharp) Lipschitz or Hoffman constants for $\mathcal{F}$ at $(b, d)$, cf. e.g. Ng and Zheng (2001), Azé and Corvellec (2002) and Zalinescu (2003). This is done in rather general settings, see also Section 2.3 of M. López' survey.

The study of uniform constants started much earlier. When looking at the recent literature, some of these early contributions seem to be forgotten. Computable formulae for Lipschitz constants for concrete pairs of norms were given e.g. by Hoffman (1952), Robinson (1973), Mangasarian (1981), Mangasarian and Shiau (1986). The important question of finding sharp estimates was first answered by $\mathrm{Li}(1993,1994)$, and this even for general pairs $\left(\|\cdot\|_{\alpha},\|\cdot\|_{\beta}\right)$ of norms.

Assuming either $C$ is of full row rank $k$ or is vacuous, $\mathrm{Li}$ (1993) shows that for any pair $\left(\|\cdot\|_{\alpha},\|\cdot\|_{\beta}\right)$,

$\lambda_{\alpha \beta}(A, C):=\sup \left\{\begin{array}{ll}u \\ v \|_{\alpha^{*}}: \begin{array}{l}\left\|A^{\top} u+C^{\top} v\right\|_{\beta^{*}}=1, u \geq 0 ; \text { the rows } \\ \text { of } A \text { related to components } u_{j}>0 \text { plus } \\ \text { the rows of } C \text { are linearly independent }\end{array}\end{array}\right\}$

is a sharp Lipschitz constant for $\mathcal{F}$, where $\|\cdot\|_{\gamma^{*}}$ denotes the dual norm to $\|\cdot\|_{\gamma}$. Note that if $\operatorname{rank}\left(\begin{array}{l}A \\ C\end{array}\right)=n$ holds and $l_{p}$ norms are used, then 
$\lambda_{\alpha \beta}(A, C)$ is given as a finite maximum of seminorms of the inverses of certain submatrices of $\left(\begin{array}{l}A \\ C\end{array}\right)$. In the 2-norm, one could get an estimate of $\lambda_{22}$ by computing the smallest eigenvalues of at most $m ! /(n-k) !(m+$ $k-n)$ ! square matrices, cf. Li (1993) for details.

Obviously, the estimates (2) and (3) are also useful for the quantitative stability analysis of the optimal set map of linear and convex quadratic programs with fixed objective function. A suitable modification of $\lambda_{\alpha \beta}(A, C)$ yields a sharp Lipschitz constant $\lambda_{\alpha \beta}(A, C, c)$ for the optimal set mapping

$$
\mathcal{F}^{o p t}(b, d)=\operatorname{argmin}\left\{c^{\top} x \mid A x \leq b, C x=d\right\},
$$

cf. Li $(1993,1994)$. Further note that the interesting question whether Lipschitz constants for $\mathcal{F}$ at $(b, d)$ are locally bounded for small perturbations of $(A, C)$ was handled first by Luo and Tseng (1994), for relations to $\lambda_{\alpha \beta}$ see also Li (1993).

Robinson (1973) considers in the case $\left[A^{\top}, C^{\top}\right] \neq O$ the constant

$$
\sigma_{\alpha \beta}(A, C):=\max _{J \subset[m]} \max _{p}\left\{\min \left\{\left\|\begin{array}{l}
u \\
v
\end{array}\right\|_{\alpha^{*}}:\left(\begin{array}{l}
u \\
v
\end{array}\right) \in S_{J}(p)\right\}: p \in P_{\beta^{*}, J}\right\},
$$

where for $J \subset[m]:=\{1, \ldots, m\}, u_{J}$ is the vector with components $u_{j}$, $j \in J, P_{\beta^{*}, J}:=\left\{p \in \mathbb{R}^{n}:\|p\|_{\beta^{*}} \leq 1, S_{J}(p) \neq \emptyset\right\}$ and

$$
S_{J}(p):=\left\{\left(\begin{array}{l}
u \\
v
\end{array}\right) \in \mathbb{R}^{m} \times \mathbb{R}^{k}: \begin{array}{l}
A^{\top} u+C^{\top} v=p \\
u_{J}=0, u \geq 0
\end{array}\right\} .
$$

He proves that $\sigma_{\alpha \beta}(A, C)$ is finite for arbitrary $\left(\|\cdot\|_{\alpha},\|\cdot\|_{\beta}\right)$ and satisfies Hoffman's estimate (3) if $\|\cdot\|_{\beta}=\|\cdot\|_{2}$ (Euclidean norm).

For any two norms $\|\cdot\|_{\alpha}$ and $\|\cdot\|_{\beta}$ and under the assumption that either $C$ has full row rank $k$ or is vacuous (put $k=0$ ), Klatte and Thiere (1996) show that

$$
\begin{aligned}
& \nu_{\alpha \beta}(A, C):=\max _{I \in \mathcal{S}} \max \left\{\left\|A^{\top} u+C^{\top} v\right\|_{\beta^{*}}-1:\left\|\begin{array}{l}
u \\
v
\end{array}\right\|_{\alpha^{*}}=1, \begin{array}{l}
u_{I} \geq 0, \\
u_{[m] \backslash I}=0
\end{array}\right\} \\
& \text { with } \mathcal{S}:=\left\{I \subset[m]:|I|=r-k, \operatorname{rank}\left[A_{I}^{\top}, C^{\top}\right]=r\right\}, r=\operatorname{rank}\left[A^{\top}, C^{\top}\right],
\end{aligned}
$$

is finite and, moreover,

$$
\lambda_{\alpha \beta}(A, C)=\nu_{\alpha \beta}(A, C)=\sigma_{\alpha \beta}(A, C) .
$$

Thus, by Li's result, all these forms give the same sharp Lipschitz constant (and uniform Hoffman constant) for $\mathcal{F}$. Note that Zalinescu's 
paper (2003) is a nice survey of more recent developments in the area of sharp global error bounds for finite linear systems.

Numerical experiments for computing sharp Lipschitz constants are not much available. In our context, Klatte and Thiere (1995) discuss several concrete representations of $\nu_{\alpha 2}(A, C)$, i.e., for $\|\cdot\|_{\beta}=\|\cdot\|_{2}$. In particular, one derives for $\|\cdot\|_{\alpha}=\|\cdot\|_{\infty}$ (maximum norm) and $\|\cdot\|_{\beta}=\|\cdot\|_{2}$ that after some transformations the computation of $\nu_{\infty 2}$ consists in solving a finite number of special quadratic programs of the form

$$
\min \left\{\|z\|_{2}: z \in \operatorname{conv}\left[\left\{A_{i}\right\}_{i \in I} \cup\left\{ \pm C_{j}\right\}_{j \in[k]}\right]\right\},
$$

where $A_{i}$ and $C_{j}$ are the rows of $A$ and $C$. Thiere's tests done in the late 1980ies, see Klatte and Thiere (1995), were never repeated by using more recent generations of solvers. Since the computable formulae for exact Lipschitz bounds under metric regularity (see the author's Section 2.3 and e.g. Canovas et al. (2008)) involve similar subproblems like $\nu_{\alpha \beta}(A, C)$, a continuation of numerical studies could be useful also for local bounds.

\section{Calmness of systems of infinitely many constraints}

In Section 2.3 the author refers in a few places to local error bounds according to definition (1.3) in his paper. We will use here the equivalent notion of calmness (in the literature also called metric subregularity) for the corresponding multifunction.

Let us consider the solution set mapping of a system of infinitely many inequalities

$$
\mathcal{F}(b)=\left\{x \in X \mid\left\langle a_{t}, x\right\rangle \leq b_{t}, t \in T\right\},
$$

where $T$ is a compact Hausdorff space, $X$ is a Banach space, $a \in \mathcal{C}(T, \mathbb{R})$ is given, while the parameter $b$ varies over $\mathcal{C}(T, \mathbb{R})$. In the author's terminology, this is the constraint set map of a continuous LIP with respect to right-hand side perturbations. The multifunction $\mathcal{F}$ is called calm at $(\bar{b}, \bar{x}) \in \operatorname{gph} \mathcal{F}$ if there exist neighborhoods $U$ and $V$ of $\bar{b}$ and $\bar{x}$, respectively, and a constant $\kappa>0$ such that

$$
d(x, \mathcal{F}(\bar{b})) \leq \kappa\|b-\bar{b}\| \quad \forall b \in U \forall x \in \mathcal{F}(b) \cap V .
$$

By definition, the multifunction $\mathcal{F}$ is calm at $(\bar{b}, \bar{x}) \in \operatorname{gph} \mathcal{F}$ if $\mathcal{F}$ is Lipschitz-like at $(\bar{b}, \bar{x})$. 
Though developed for more general nonlinear systems, the results in Henrion and Outrata (2005), Klatte and Kummer (2009) and Kummer (2009) have interesting implications also for the setting (6). Fixing $\bar{b} \in$ $\mathcal{C}(T, \mathbb{R})$, we write for $x \in X$

$$
g_{t}(x):=\left\langle a_{t}, x\right\rangle-\bar{b}_{t}, t \in T, \quad g(x):=\max _{t \in T} g_{t}(x),
$$

and define the relative slack of $g_{t}$ in comparison with $g$ for all $x \in X$ with $g(x)>0$ by

$$
s_{t}(x)=\frac{g(x)-g_{t}(x)}{g(x)} \quad(\geq 0), t \in T .
$$

Theorem 3 by Klatte and Kummer (2009) implies the following characterization of calmness for $\mathcal{F}$ : Let $\bar{x}$ be any point such that $g(\bar{x})=0$, i.e., $\left\langle a_{t}, \bar{x}\right\rangle \leq \bar{b}_{t}$ for all $t$ in $T$, and there is some $s \in T$ with $\left\langle a_{s}, \bar{x}\right\rangle=\bar{b}_{s}$. Then $\mathcal{F}$ is calm at $(\bar{b}, \bar{x})$ if and only if there exist some $\lambda \in(0,1)$ and a neighborhood $\Omega$ of $\bar{x}$ such that the following holds:

for all $x \in \Omega$ with $g(x)>0$ there is some $u$

$$
\text { satisfying }\|u\|=1 \text { and }\left\langle a_{t}, u\right\rangle \leq \frac{s_{t}(x)}{\lambda}-\lambda \quad \forall t \in T \text {. }
$$

For $X=\mathbb{R}^{n},(8)$ differs from the extended Mangasarian-Fromovitz constrain qualification (EMFCQ) - which characterizes the Lipschitz-like property - just by the additionally appearing quantities $s_{i}(x)$. In Klatte and Kummer (2009) and Kummer (2009) this result is used for characterizing calmness by uniform - with respect to certain starting points linear convergence of suitable descent methods. This is of interest in developing solution methods for solving equations or variational problems which require calmness only.

In their Theorem 4, Henrion and Outrata (2005) give a sufficient condition for calmness of the solution set map of an inequality system defined by infinitely many differentiable functions, but for finitedimensional $X$ and $T$. This condition lies between EMFCQ and the extended Abadie constraint qualification, it is written in terms of a family of so-called critical index sets defined as Hausdorff limits of sequences of active index sets. It directly applies also to the setting (6).

\section{References}

Azé D. and Corvellec J.-N. (2002). On the sensitivity analysis of Hoffman constants for systems of linear inequalities, SIAM J. Optim. 12, 913-927. 
Cánovas M.J., Gómez-Senent F.J., Parra J. (2008). Regularity modulus of arbitrarily perturbed linear inequality systems. J. Math. Anal. Appl., 343, 315-327.

Henrion R. and Outrata J. V. (2005). Calmness of constraint systems with applications. Math. Program., Ser. B, 104, 437-464.

Hoffman A.J. (1952). On approximate solutions of systems of linear inequalities. J. Res. Nat. Bur. Standards 49, 263-265.

Klatte D. and Kummer B. (2009). Optimization methods and stability of inclusions in Banach spaces. Math. Program. Ser. B, 117, 305-330.

Klatte D. and Thiere G. (1995). Error bounds for solutions of linear equations and inequalities. Z. Oper. Res. 41, 191-214.

Klatte D. and Thiere G. (1996). A note on Lipschitz constants for solutions of linear inequalities and equations. Linear Algebra Appl. 244, 365-374.

Kummer B. (2009). Inclusions in general spaces: Hoelder stability, solution schemes and Ekeland's principle. J. Math. Anal. Appl., 358, 327-344.

Li W. (1993). The sharp Lipschitz constants for feasible and optimal solutions of a perturbed linear program. Linear Algebra Appl. 187, 15-40.

Li W. (1994). Lipschitz constants for basic optimal solutions and basic feasible solutions of linear programs. SIAM J. Control Optim. 32, 140-153.

Luo Z.Q. and Tseng P. (1994). Perturbation analysis of a condition number for linear systems. SIAM J. Matrix Anal. Appl. 15, 636-660.

Mangasarian O.L. (1981). A condition number of linear inequalities and equalities. Methods Oper. Res. 43, 3-15.

Mangasarian O.L. and Shiau T.H. (1986). Lipschitz continuity of solutions of linear inequalities, programs and complementarity problems. SIAM J. Control Optim. 25, 583-595.

Ng K.F. and Zheng X.Y. (2001) On error bounds for lower semicontinuous functions, SIAM J. Optim. 12, 1-17.

Robinson S.M. (1973) Bounds for error in the solution set of a perturbed linear program. Linear Algebra Appl., 6, 1973.

Zalinescu C. (2003) Sharp estimates for Hoffman's constant for systems of linear inequalities and equalities, SIAM J. Optim. 14, 517-533. 